\title{
Simple and safe bedside method for serial measurement of left ventricular ejection fraction, cardiac output, and pulmonary blood volume ${ }^{1}$
}

\author{
Peter P. Steele, Donald Van Dyke, Richard S. Trow, Hal O. Anger, and Hywel Davies \\ From the Division of Cardiology, University of Colorado Medical Center, Denver, Colorado; Section of Cardi- \\ ology and Division of Nuclear Medicine, Veterans Administration Hospital, Denver, Colorado; and the Donner \\ Laboratories, University of California, Berkeley, California, U.S.A.
}

A simple, safe method for bedside measurement of left ventricular function and pulmonary blood volume has been developed. A single scintillation probe positioned over the mid-left ventricle records the passage of bolus of radionuclide ${ }^{113 \mathrm{~m}}$ In) injected into the superior vena cava, from which the left ventricular ejection fraction can be determined after proper background correction. ${ }^{113 \mathrm{~m}}$ In, half-life $I \cdot 7$ hours, easily prepared from a commercially available generator, rapidly binds to plasma transferrin and can be used to determine blood volume and cardiac output. With cardiac output and left ventricular ejection fraction determined simultaneously, left ventricular end-diastolic volume can be calculated and pulmonary blood volume can be determined from pulmonary transit time and cardiac output.

With this method, we have demonstrated stable and changing left ventricular function and pulmonary blood volume in a variety of clinical situations, e.g. myocardial infarction, pulmonary embolism, and severe pulmonary parenchymal disease. Blood volume and cardiac output so determined correlate well with standard techniques. The left ventricular ejection fraction determined by this method correlates well with this measurement obtained from left ventricular angiography. Loss of the pulmonary valley between the right and left ventricles has been demonstrated in severe pulmonary vascular obstruction (embolism), in severe bronchial obstruction, and as a result of massive right ventricular dilatation in the presence of normal pulmonary blood volume. This method, which can be frequently performed, has proved useful in serial evaluation of cardiac function at the bedside.

The ability to quantitate left ventricular function at the bedside would be an important aid for the care of the seriously ill patient, especially if the measurement could be repeated frequently. Not only would such information be of value in the management of the individual patient, but it would provide the basis for evaluation of various forms of therapy. External monitoring of the passage of a bolus of radioactive tracer through the central circulation has been developed over the past 25 years (Prinzmetal et al., 1948) in an attempt to fulfil the need for a safe, easily repeatable method of quantitating cardiac function. Earlier attempts to use simple scintillation probes and low frequency data for evaluation of the left ventricle after a right-sided Received 28 August 1973.

1 Supported in part by Veterans Administration Research Funds and U.S. Atomic Energy Commission Contract. injection failed because of the difficulties in analysing the complicated functions resulting from the passage of the bolus through the right heart chambers and lungs (Bianchi et al., I96I; Donato, 1962; Sullivan et al., I97I ; Van Dyke et al., 1972; Weber, dos Remedios, and Jaski, 1972).

With the advent of scintillation cameras with high frequency recording, interfaced to data storage, acquisition, and manipulation systems a better understanding of the influence of ventricular function on the radioisotope concentration patterns has been obtained. What has been learned is that, though the low frequency patterns in the left ventricle, after an injection on the right side, have not as yet provided clinically useful information about the left ventricle, high frequency recording of count rate from within the left ventricle, when appropriately corrected for counts from surrounding tissue, 
provides an accurate measure of left ventricular ejection fraction (Van Dyke et al., 1972; Weber et al., 1972). This information, combined with the classic information obtainable from the low frequency part of the data (cardiac output and pulmonary blood volume), provides an abundance of clinically useful information.

Since it is not easy to locate the scintillation camera and its associated data storage system at the patient's bedside, the use of an easily portable, properly collimated single probe with direct strip chart recording has been investigated. The technique requires determining the mid-point of the left ventricle from a chest film, the introduction of a central venous catheter, and recording first from the left ventricle and subsequently from surrounding tissues after eclipsing the left ventricle. Results from the serial study of patients with stable or changing cardiac and pulmonary function are presented.

The single probe praecordial radioisotope content curve consists of II parts, each of which provides evidence of central circulatory function (Fig. I).

\section{Methods}

In order to measure blood volume (which is required for calculating cardiac output by the externally monitored radioisotope method) one must inject agents such as ${ }^{99 \mathrm{~m}}$ Technetium-albumin (Benjamin, Rejali, and Friedell, 1970) or ionic ${ }^{113 \mathrm{~m}}$ Indium, which remain within the vascular compartment. When ${ }^{113 m}$ In-chloride (in gelatin solution) is injected intravenously, ${ }^{113 \mathrm{~m}}$ In binds immediately to the plasma transferrin (Hosain et al., 1969; Stern et al., 1967). Because no preparation time is required for ${ }^{113 \mathrm{~m}} \mathrm{In}$ and because its energy is suitable for use with the probe, this short-lived (I $\cdot 7$-hour halflife) radionuclide, which is obtained from a long-lived generator (II8 days) has been used in the majority of the studies reported here.

We have estimated the radiation dose resulting from the administration of $\mathrm{r} \cdot 5 \mathrm{mCi}$ of ${ }^{113 \mathrm{~m}} \mathrm{In}$ to be $320 \mathrm{mrads}$ to the blood and 30 mrads to the whole body.

Preparation of the patient for study requires inserting a catheter to the superior vena cava, taping a radioopaque marker, such as a paper clip, to the skin over the estimated mid-point of the left ventricle, and then taking a portable $x$-ray in the supine position. From the $x$-ray the position of the catheter in the superior vena cava, and the mid-point of the left ventricle relative to the



FIG. I I) Overall transit time through the central circulation (time from injection to peak of $L V)$ : prolonged in circulatory failure. 2) Rate of appearance of label in right ventricle $(R V)($ flow from central vein to $R V$ ): prolonged in circulatory failure. 3) Downslope of $R V$ washout curve: slow in circulatory failure and in the presence of dilated right heart chambers. 4) Pulmonary transit time decreased with decreased pulmonary blood volume $(P B V)$ and vice versa: decreased in right-to-left shunt and increased in circulatory failure. 5) Temporal separation between $R V$ and $L V$ : obliterated with much decreased $P B V$ or very dilated right heart chambers. 6) Area under the first pass of the indicator: when related to the concentration after mixing in the blood, No. II, is inversely related to cardiac output. 7) Counts from surrounding tissues after eclipsing the $L V$ : provides the baseline against which EF is measured. 8) Ejection fraction (EF): related to No. 7. 9) Washout slope of LV: slow in failure. 10) First recirculation: shortened in arteriovenous fistula and blunted in circulatory failure. II) Concentration after complete mixing: small (as compared to area under first pass, No. 6) in low cardiac output or exceptionally large blood volume. 
marker can be confirmed. The correct position for the probe is then drawn on the chest with a felt pen to facilitate accurate repositioning for serial studies.

A standard, commercially available, portable scintillation probe with a $5 \mathrm{~cm}$ by $5 \mathrm{~cm}$ sodium iodide crystal is used. This portable stand carries the high voltage power supply, count rate meter with ro million counts/min capacity and time constant of less than $0.1 \mathrm{sec}$, and high speed strip chart recorder. Linearity of the entire system is demonstrated by rotating an absorber wheel with ro steps having from 0 to roo per cent absorption over a I $\mathrm{mCi}{ }^{113 \mathrm{~m}}$ In source.

Collimation for obtaining the left ventricular radioisotope count rate curve is shown at the top of Fig. 2. A $3.5 \mathrm{~cm}$ circular port (in $1.3 \mathrm{~cm}$ lead) collimator is positioned over the mid-point of the left ventricle. A bolus of $\mathrm{I} \mathrm{mCi}{ }^{113 \mathrm{~m}} \mathrm{In}$ is flushed through the central venous catheter and the activity over the left ventricle recorded with the probe perpendicular to the chest wall. Recording is repeated after 5 minutes to obtain the concentration after mixing. A blood sample is taken at this time for determination of blood volume. From these two measurements, cardiac output is determined.

Collimation of the probe is then modified to the configuration shown at the bottom of Fig. 2 for recording activity immediately surrounding the left ventricle. The left ventricle is eclipsed by a $5.5 \mathrm{~cm}$ circular shield placed $3.5 \mathrm{~cm}$ out from the probe face $(1 \mathrm{I} .6 \mathrm{~cm}$ from the sodium iodide crystal) and maintained in position by a styrofoam cone. For this recording a second bolus of $500 \mu \mathrm{Ci}^{113 \mathrm{~m}} \mathrm{In}$ is injected. The tails of the two records are matched by holding the two translucent records against an illuminated $x$-ray viewing box, and the mean background curve is traced on the left ventricular curve. From these the ejection fraction is calculated. The rationale and method for this correction have been presented in detail elsewhere (Van Dyke et al., 1972).

The pulmonary blood volume is obtained from the product of the cardiac output and pulmonary transit time using a modification of the formulation of Giuntini et al. (1963). The pulmonary transit time is estimated as the interval from the time of ejection of label from the right ventricle ( 75 per cent of peak value) to the time of peak count rate in the left heart. The basis of the modification used in this study is described below under low frequency events.

\section{Results}

\section{Normal praecordial curves}

Fig. I and 2 show characteristic radioisotope content curves from the mid left ventricular praecordial position in the presence of normal cardiac function. The first peak represents passage of the bolus through the right ventricle; the first valley (the pulmonary valley) its passage out of the field of view of the probe into the pulmonary circulation; and the second peak the left ventricular portion of the curve with the ejection fraction superimposed. The corrected baseline from which the ejection fraction is measured is shown as a smooth curve under the left ventricular washout portion of the record.
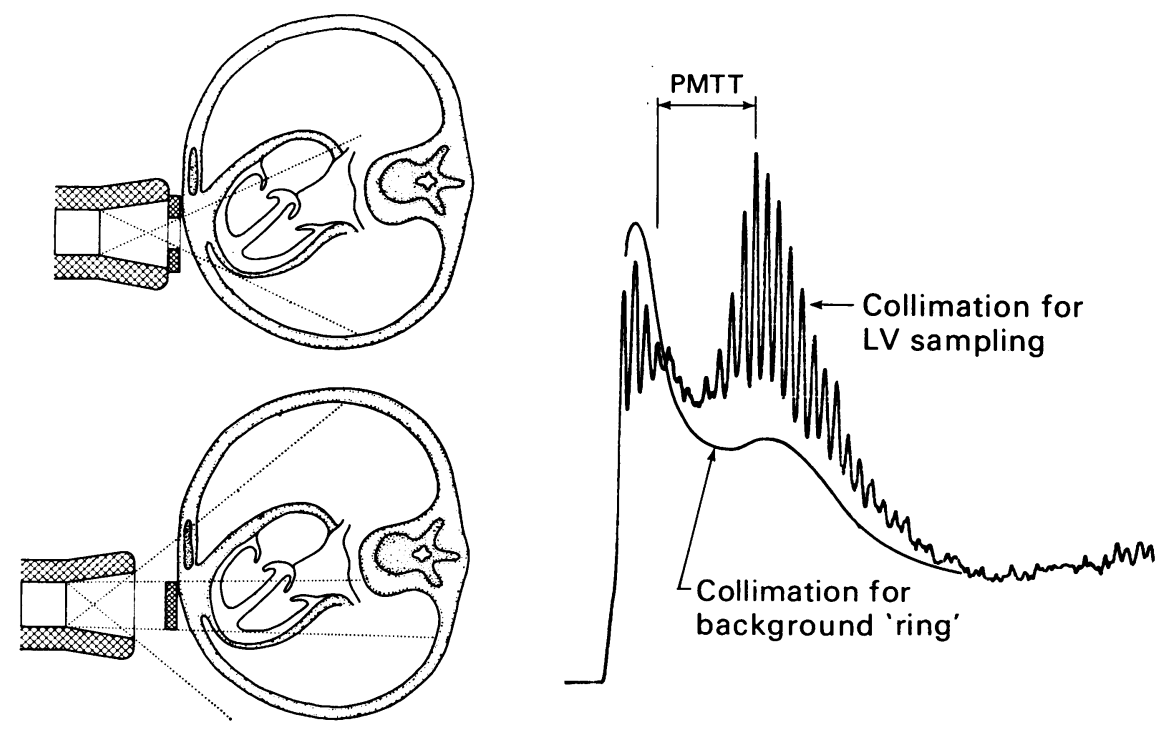

DBL 7212-5005

FIG. 2 The collimation for obtaining the $L V$ radioisotope content curve (top) and eclipse collimation for obtaining 'cross talk' correction (bottom). The auxillary collimators are made of $\mathrm{I} / 2$ in. lead. 


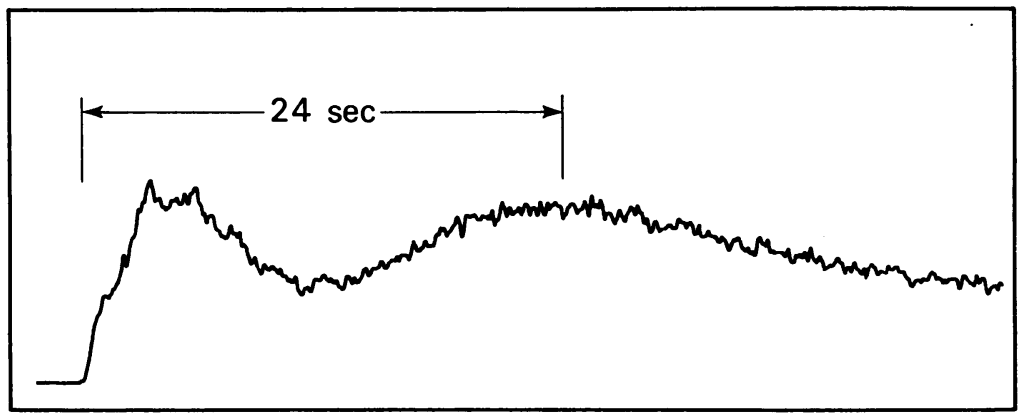

DBL 72115578

FIG. 3 A grossly abnormal pattern (prolonged transit time and loss of LVEF) obtained shortly before death from a massive myocardial infarct.


Isoprenaline dependence

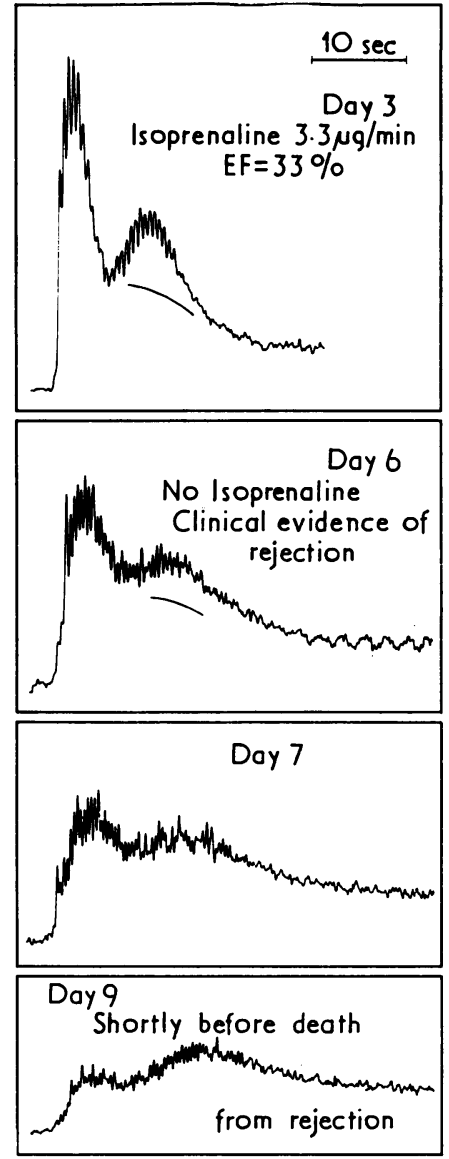

Rejection

FIG. 4 Representative patterns from 21 serial studies done over a 7-day period in the recipient of a heart transplant. The records demonstrate the effects of surgical trauma (top left), drugs (bottom left), and rejection (right) (see text). 


\section{Low frequency events}

An important qualitative aspect of the record is overall transit time through the central circulation which is most easily represented by the time elapsing between injection into the superior vena cava and peak activity in the left ventricle as illustrated in Fig. 3 and 4. Fig. 3 shows a grossly abnormal pattern obtained shortly before death in a patient with a massive myocardial infarct. The possibility of the sudden onset of respiratory distress being due to pulmonary embolus was a real one. The radioisotope record was clearly that of myocardial dysfunction rather than massive pulmonary embolism (see below).

Fig. 4 shows representative patterns from $2 \mathrm{I}$ serial studies performed over a 7-day period in the recipient of a heart transplant. Left ventricular dysfunction is apparent in the much prolonged transit time in the record obtained 3 hours after completion of the surgical procedure. Dependence of the newlytransplanted heart on isoprenaline is apparent in the 4 subsequent records. Cardiac arrest occurred shortly after the 4 th record was obtained. After resuscitation and re-initiation of a dose of $\mathrm{IO} \mu \mathrm{g} / \mathrm{min}$ of isoprenaline an entirely normal pattern with ejection fraction of 69 per cent and overall transit time of 7 seconds was recorded. From the 2nd to the 7th day, when cardiac arrest and death occurred due to rejection of the heart, deterioration was progressive (overall transit time of 16 seconds on day 7).

Transit time through the central circulation is related to cardiac output and volume of blood in the various compartments of the central circulation, as discussed later.

Normally, between the right and left ventricular peaks of the radiocardiogram there is a valley (the pulmonary valley) during which the label is in the lungs. This results in temporal separation of the right and left ventricle (Fig. I).

The record from a patient in severe respiratory distress from multiple pulmonary thromboemboli is shown in Fig. 5. Part A of the figure shows complete loss of the pulmonary valley (temporal separation between right and left ventricle). Introduction of the label into the pulmonary artery (shaded curve of part B) resulted in abnormally rapid labelling of the left ventricle. Fig. 6 shows the gradual emergence of temporal separation with time in the same patient. The method of Giuntini et al. (1963) for calculation of pulmonary blood volume uses the value of 37 per cent of the right ventricular peak count as representing the mean time spent in the right ventricle. Our studies have shown that this figure is not universally applicable, for it would


DBL 731-5018

FIG. 5 A) An abnormal pattern obtained when the probe was positioned over the $L V$ of a patient subsequently shown to have multiple pulmonary emboli. The reduced pulmonary blood volume obscured temporal separation of right and left ventricle. $B$ ) The pattern obtained with the probe over the RV after an SVC injection superimposed on the pattern of LV after injection into the pulmonary artery by means of a flow-directed catheter. 

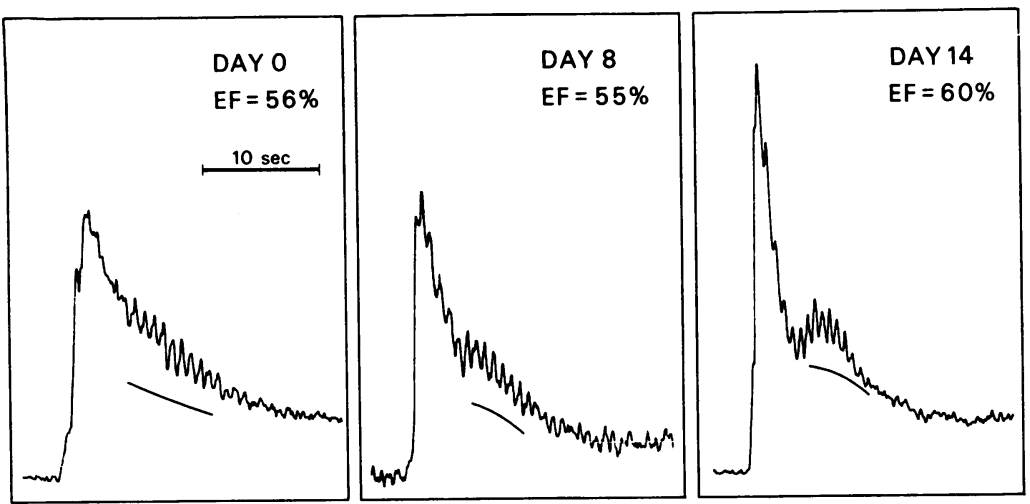

FIG. 6 The gradual emergence of the pulmonary valley between $R V$ and $L V$ with time in the same patient illustrated in Fig. 5.
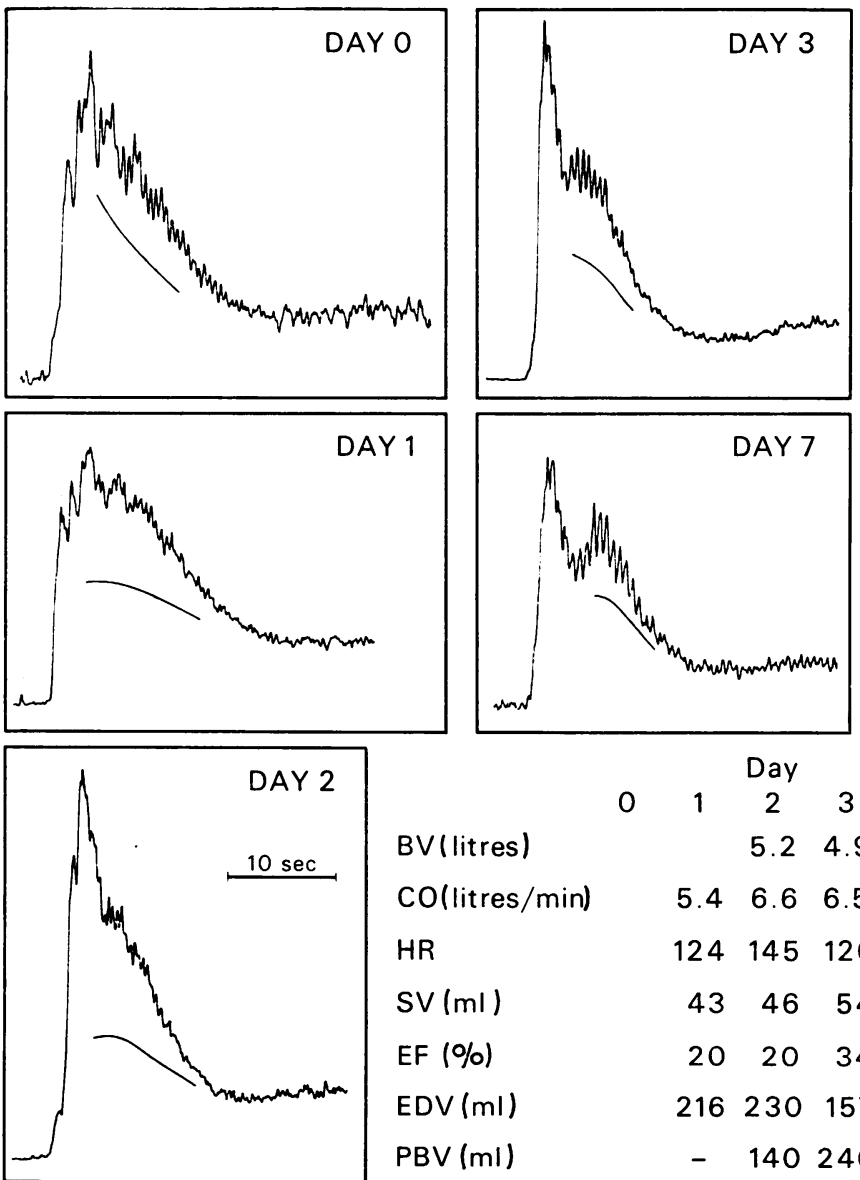

\begin{tabular}{lcrccc} 
& \multicolumn{5}{c}{ Day } \\
& 0 & 1 & 2 & 3 & 7 \\
BV(litres) & & 5.2 & 4.9 & \\
CO(litres/min) & 5.4 & 6.6 & 6.5 & 4.9 \\
HR & 124 & 145 & 120 & 96 \\
SV $(\mathrm{ml})$ & 43 & 46 & 54 & 51 \\
EF $(\%)$ & 20 & 20 & 34 & 62 \\
EDV $(\mathrm{ml})$ & 216 & 230 & 157 & 83 \\
PBV $(\mathrm{ml})$ & - & 140 & 240 & 245
\end{tabular}

FIG. 7 A series of studies from a patient with initial loss and gradual reappearance of the pulmonary valley between $R V$ and $L V$. This patient had severe respiratory distress caused by widespread bronchial carcinoma complicated by pneumonia.

$B V=$ Blood volume: $C O=$ Cardiac output $: H R=$ Heart rate: $S V=$ Stroke volume: $E F=$ Ejection fraction: $E D V=$ End-diastolic volume: $P B V=$ Pulmonary blood volume. 
result in the right ventricular registration occurring after that of the left ventricle, as for example in Fig 6. A reasonable minimal value $(100-200 \mathrm{ml})$ for pulmonary blood volume was obtained in this case only by using the figure of 70 or 80 per cent of peak right ventricle instead of the 37 per cent of Giuntini.

It, therefore, appears to be reasonable that the figure of 75 per cent rather than 37 per cent should be employed for this calculation in all cases. In normal subjects the fall of right ventricular activity is so rapid that it makes little practical difference which of these values is adopted.

Fig. 7 shows a series of studies from another patient with initial loss and gradual reappearance of temporal separation between the right and left ventricle. This patient had severe respiratory distress due to widespread bronchial carcinoma complicated by pneumonia.

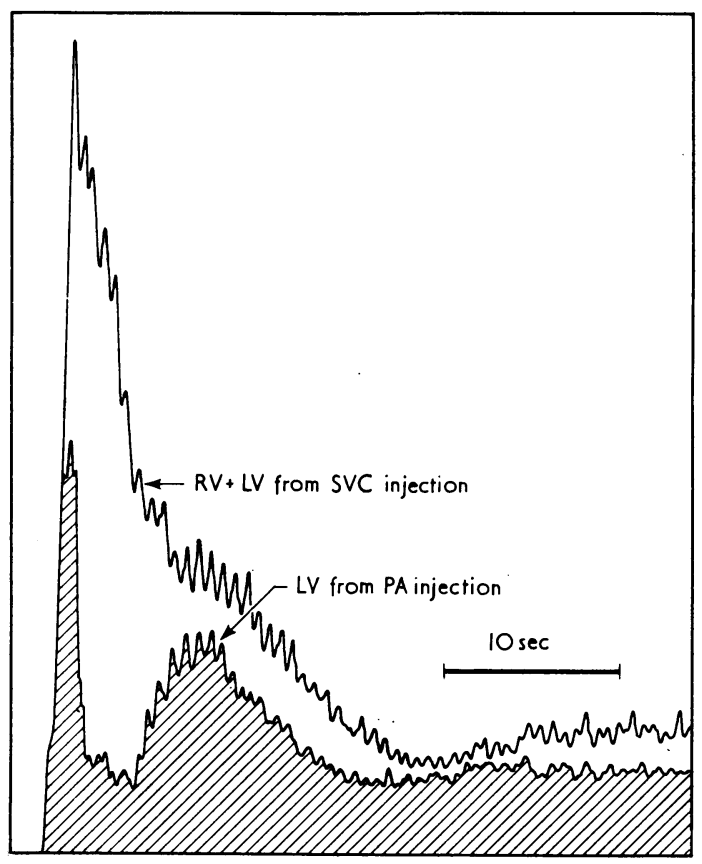

FIG. 8 Another mechanism by which the pulmonary valley between $R V$ and $L V$ may be obliterated. In this case the PTT was normal and loss of the valley was due to massive dilatation of right heart chambers. The shaded portion (bottom) of the figure shows the pattern obtained over the $L V$ when the radioisotope was introduced into the pulmonary artery, bypassing the dilated $R V . \quad B V=6$ litres; $C O=5.9$ litres; $H R=80 ; S V=73 \mathrm{ml}$. In the $L V, S V=73 \mathrm{ml}$; $E F=37$ per cent $; E D V=200 \mathrm{ml} ; P B V=519 \mathrm{ml}$. (Abbreviations as for Fig. 7.)
Fig. 8 illustrates another mechanism by which the pulmonary valley between right and left ventricle may be obliterated. On first examination (by pattern recognition alone) it might appear that this patient had a much reduced pulmonary blood volume. However, the pulmonary transit time was found to be $5.3 \mathrm{sec}$, or the equivalent of $7 \cdot$ I stroke volumes, giving a normal $(520 \mathrm{ml})$ pulmonary blood volume. Introduction of the label into the pulmonary artery again indicated a normal pulmonary transit time (lower curve in Fig. 8), in contradistinction to Fig. 5. Scintillation camera pictures taken during the passage of a bolus of ${ }^{99 \mathrm{~m}} \mathrm{Tc}$ through the right heart showed an extremely large right atrium and right ventricle. Thus, dilatation of the right heart chambers to volumes approaching the pulmonary blood volume will result in obliteration of the pulmonary valley in the record, but not in a decrease of the pulmonary transit time between right and left ventricle.

\section{High frequency events (left ventricular fraction)}

Ejection fraction is important for its intrinsic value as an indicator of left ventricular function (Dodge and Baxley, 1968) and as the basis for the calculation of left ventricular end-diastolic volume (net stroke volume/ejection fraction). Changes in ejection fraction with changing left ventricular function are illustrated in Fig. 3 and 4. A series of studies in a patient with congestive heart failure during alcoholic detoxication is shown in Fig. 9. As can be seen, the low frequency pattern and cardiac output were not abnormal, but the ejection fraction was conspicuously depressed with only slight improvement over the 6 days during which the patient was studied. The combination of normal cardiac output and heart rate and small ejection fraction signifies a large enddiastolic volume $(300 \mathrm{ml}$ average value for the 5 studies), interpreted in this case as being due to depressed myocardial function in a patient with alcoholic cardiomyopathy (Evans, 196I).

Fig. Io shows the striking effect of sublingual isosorbide dinitrite on left ventricular ejection fraction and end-diastolic volume in a patient with coronary artery disease. Administration of the drug produced transient, but significant changes in all parameters measured, which persisted for 90 minutes.

\section{Discussion}

It is necessary to appreciate that the high-frequency variation in the count rate is an expression of content rather than concentration in the cardiac chambers. It became apparent as these studies progressed that 



$\begin{array}{lrrrrr}\text { BV (litres) } & 7.5 & 6.8 & 6.8 & & \\ \text { CO (litres/min) } & 6.4 & 7.8 & 9.0 & 7.9 & 7.4 \\ \text { HR } & 100 & 88 & 96 & 88 & 84 \\ \text { SV (ml) } & 64 & 88 & 94 & 90 & 88 \\ \text { EF (\%) } & 25 & 25 & 25 & 34 & 33 \\ \text { EDV (ml) } & 256 & 350 & 377 & 260 & 270 \\ \text { PBV (ml) } & 640 & 640 & 660 & 585 & 660\end{array}$

FI G. 9 A series of studies done during alcoholic detoxification in a man with congestive failure. This series illustrates the stability of the PBV measurement in a patient whose difficulties resulted from $L V$ dysfunction. (Abbreviations as for Fig. 7.)

in viewing sequential recordings it is possible to recognize improvement or deterioration of cardiac function visually from the pattern alone. Measurements of ejection fraction and transit times provide an easy method of quantitation, thus adding significantly to the value of the data though rarely changing the impression gained from the first viewing of the pattern characteristics of the strip chart recording. Thus, the method has the singular advantage of permitting almost immediate evaluation of cardiac function. This compares favourably to the considerable delay when a scintillation camera able to store, to retrieve, and manipulate data is used.

If in a given case information is required on cardiac output, left ventricular end-diastolic volume, and pulmonary blood volume, such information can be obtained using the single probe and ${ }^{99 \mathrm{~m}} \mathrm{Tc}-$ albumin or ionic ${ }^{113 \mathrm{~m}} \mathrm{In}$. This greater information is obtained at the cost of greater complexity (preparation of the labelled albumin or elution of the In generator, blood samples, preparation of a standard, sample counting, and computation time).

Experience with more elaborate systems (Van Dyke et al., 1972; Weber et al., 1972) indicated that a single count rate curve obtained over the left ventricle would be useful provided that a satisfactory baseline correction could be achieved. Could a fixed background ring (eclipse collimation of the left ventricle) serve to provide the proper correction for all subjects in all situations? The eclipse collimation used in these studies appears to be surprisingly satisfactory over a wide range of ejection fraction (17-87 per cent) when compared to standard angiography (Fig. II).

In these patients left ventricular angiograms were filmed using standard techniques in the $40^{\circ}$ right 
Before sorbide nitrote



3 min after sorbide nitrate

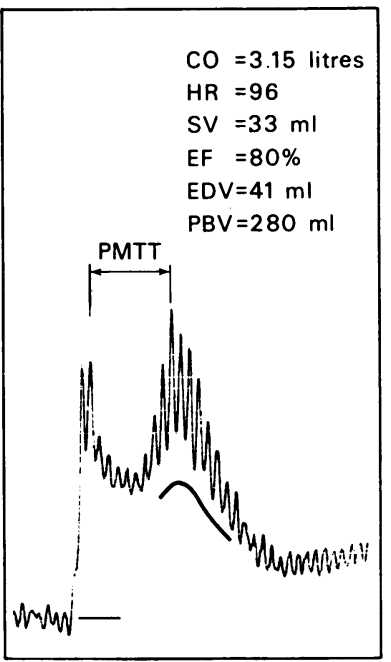

After sorbide nitrate

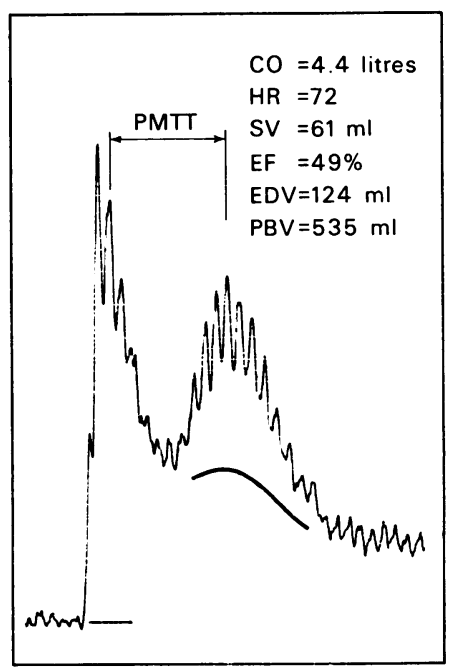

FIG. IO A striking effect of isosorbide dinitrite administered sublingually, on LVEF and EDV in a patient with coronary artery disease.

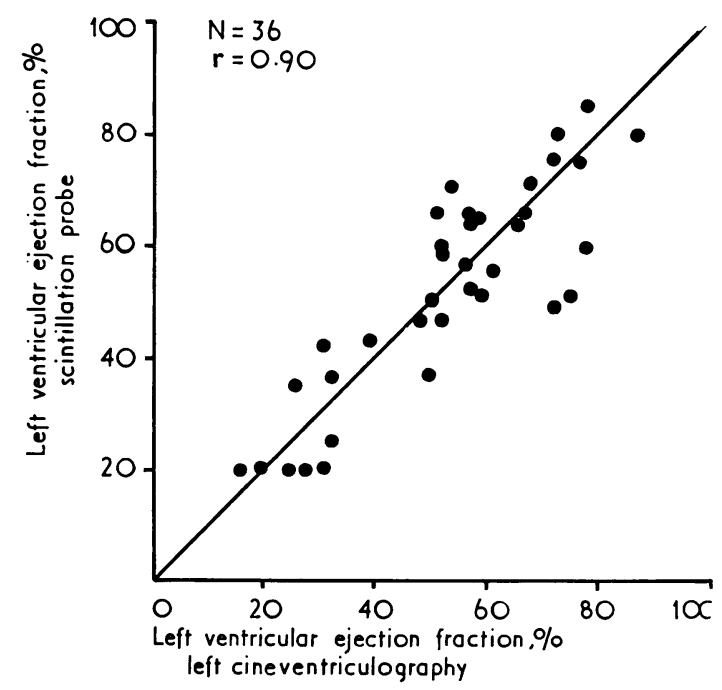

FIG. I I The correlation of LVEF measured with the scintillation probe and with left cine-ventriculography.

anterior oblique position and left ventricular volumes were obtained by area length computation with correction for magnification (Dodge et al., 1960).

Note that in the last 2 records of the series shown in Fig. 4, ejection fraction could not be estimated. This was because as rejection and left ventricular dysfunction progressed, both left ventricular and background configurations resulted in similar patterns during the left ventricular phase of the record because of pronounced dilatation of the left ventricle (central port and eclipse ring seeing the same information). However, before this becomes a problem, transit time has become much prolonged and the ejection fraction has fallen below clearly recognizable amplitude, so that the need for a baseline against which to judge the ejection fraction has disappeared.

Accurate measurement of left ventricular ejection fraction requires precise placement of the probe over the mid-point of the left ventricle. Even small errors in probe position can result in false increase or decrease in recorded left ventricular ejection fraction.

Except for its use in transit time analysis and in cases where gross abnormality imposes itself on the record (Fig. 8), no attempt has been made to quantitate the right ventricle in these studies. Previous studies (Prinzmetal et al., 1948; Bianchi et al., 196I ; Donato, 1962; Sullivan et al., I971 ; Van Dyke et al., 1972; Weber et al., 1972; Folse and Braunwald, I962) have shown that determination of right ventricular ejection fraction will depend on adequate correction of the praecordial record for mixing, streaming, and counts from surrounding tissues. Whether the eclipse mask technique applied in these studies to the left ventricle would be equally applicable to the right ventricle is under investigation. 
The time required for the study as well as the dose of radioisotope to the patient could be reduced if the left ventricular and left ventricular eclipse recordings could be made simultaneously. A dual probe with dual count rate meters and dual channel pen recorder is being built for this purpose.

Hosain et al. (1969) have shown that blood volume can be accurately measured with $113 \mathrm{~m}$ In, since this radionuclide rapidly binds to plasma transferrin. We have measured blood volume in 9 patients with both ${ }^{113 \mathrm{~m}} \mathrm{In}$ and radioiodinated $\left({ }^{125} \mathrm{I}\right)$ serum albumin with good correlation $(r=0.80)$. Rarely, the value for blood volume measured with ${ }^{113 \mathrm{~m} I n}$ yields an absurdly high value (e.g. I4 litres). This is easily recognized because it is so far from the expected value. This has occurred in desperately ill patients all of whom have died. The mechanism is not at present understood.

Cardiac output was measured in 35 patients with ${ }^{113 m} \mathrm{~m}$ and the scintillation probe and with indiocyanine green dye (injection into pulmonary artery, sampling from ascending aorta or brachial artery) with good correlation $(r=0.78)$.

The portable scintillation probe should prove useful for bedside evaluation of cardiopulmonary function in the seriously ill. In particular this technique should help to define the abnormalities present in cases of circulatory collapse, where the diagnostic considerations are major pulmonary embolism and myocardial infarction with left ventricular dysfunction. Shortened pulmonary transit time suggests pulmonary embolism and is easily and rapidly distinguished from the long transit time and reduced left ventricular ejection fraction of major left ventricular dysfunction.

We would like to thank Drs. T. E. Starzl and G. Pappas for permission to study the patient illustrated in Fig. 4, and Dr. R. W. Sullivan (USPHS Hospital, San Francisco) for preliminary clinical evaluation of the probe collimation.

\section{References}

Benjamin, P. P., Rejali, A., and Friedell, H. (I970). Electrolytic complexation of ${ }^{99 \mathrm{~m}} \mathrm{Tc}$ at constant current: its applications in nuclear medicine. Fournal of Nuclear Medicine, II, 147.

Bianchi, R., Cerri, B., Giuntini, C., and Toni, P. (I96I). Validita e limiti del radiocardiogramma da iniezione giugulare di Sari. Minerva Nucleare, 5, 125. (Available in English translation as $U C R L-T$ rans. I482.)

Dodge, H. T., and Baxley, W.A. (I968). Hemodynamic aspects of heart failure. American fournal of Cardiology, 22, 24.

Dodge, H. T., Sandler, H., Ballew, D. W., and Lord, J. D. (I960). The use of biplane angiocardiography for the measurement of left ventricular volume in man. American Heart fournal, 60, 762.

Donato, L. (1962). Selective quantitative radiocardiography. Progress in Cardiovascular Diseases, 5, I.

Evans, W. (I96I). Alcoholic cardiomyopathy. American Heart fournal, 61, 556.

Folse, R., and Braunwald, E. (1962). Determination of fraction of left ventricular volume ejected per beat of ventricular end-diastolic and residual volumes. Circulation, 25, 674 .

Giuntini, C., Lewis, M. L., Sales Luis, A., and Harvey, R. M. (1963). A study of the pulmonary blood volume in man by quantitative radiocardiography. Fournal of Clinical Investigation, 42, 1589.

Hosain, P., Hosain, F., Iqbal, Q. M., Carulli, N., and Wagner, H. N. (1969). Measurement of plasma volume using ${ }^{99 m} \mathrm{Tc}$ and ${ }^{113 m}$ In labeled proteins. British fournal of Radiology, $42,627$.

Prinzmetal, M., Corday, E., Bergman, H. C., Schwartz, L., and Spritzler, R. J. (1948). Radiocardiography: a new method for studying the blood flow through the chambers of the heart in human beings. Science, 108, 340.

Stern, H. S., Goodwin, D. A., Scheffel, U., Wagner, H. N., and Kramer, H. H. (1967). ${ }^{113 \mathrm{~m}}$ In for blood pool and brain scanning. Nucleonics, $25,62$.

Sullivan, R. W., Bergeron, D. A., Vetter, W. R., Hyatt, K. H., Haughton, V., and Vogel, J. M. (197I). Peripheral venous scintillation angiocardiography in determination of left ventricular volume in man. American fournal of Cardiology, $28,563$.

Van Dyke, D., Anger, H. O., Sullivan, R. W., Vetter, W. R., Yano, Y., and Parker, H. G. (1972). Cardiac evaluation from radioisotope dynamics. Fournal of Nuclear Medicine, 13, 585 .

Weber, P. M., dos Remedios, L. V., and Jaski. I. A. (1972). Quantitative radioisotopic angiocardiography. Fournal of Nuclear Medicine, 13, 815.

Requests for reprints to Dr. Peter Steele, Denver V. A. Hospital, ro55 Clermont Avenue, Denver, Colorado 80220 , U.S.A. 